\title{
Development of an epitope conservancy analysis tool to facilitate the design of epitope-based diagnostics and vaccines Huynh-Hoa Bui1 ${ }^{1,2}$, John Sidney ${ }^{1}$, Wei Li ${ }^{1}$, Nicolas Fusseder ${ }^{1}$ and Alessandro Sette*1
} \author{
Pharmaceuticals, Inc., Antisense Drug Discovery, 1896 Rutherford Road, Carlsbad, CA 92008, USA \\ Email: Huynh-Hoa Bui - hbui@isisph.com; John Sidney - jsidney@liai.org; Wei Li - weilee.li@gmail.com; \\ Nicolas Fusseder - fusseder@gmail.com; Alessandro Sette* - alex@liai.org \\ * Corresponding author
}

Address: ${ }^{1}$ La Jolla Institute for Allergy and Immunology, Division of Vaccine Discovery, 9420 Athena Circle, La Jolla, CA 92037, USA and 2 Isis

Published: 26 September 2007

BMC Bioinformatics 2007, 8:361

This article is available from: http://www.biomedcentral.com/|47|-2/05/8/36|

(C) 2007 Bui et al; licensee BioMed Central Ltd.

This is an Open Access article distributed under the terms of the Creative Commons Attribution License (http://creativecommons.org/licenses/by/2.0), which permits unrestricted use, distribution, and reproduction in any medium, provided the original work is properly cited.
Received: I March 2007

Accepted: 26 September 2007

\begin{abstract}
Background: In an epitope-based vaccine setting, the use of conserved epitopes would be expected to provide broader protection across multiple strains, or even species, than epitopes derived from highly variable genome regions. Conversely, in a diagnostic and disease monitoring setting, epitopes that are specific to a given pathogen strain, for example, can be used to monitor responses to that particular infectious strain. In both cases, concrete information pertaining to the degree of conservancy of the epitope(s) considered is crucial.
\end{abstract}

Results: To assist in the selection of epitopes with the desired degree of conservation, we have developed a new tool to determine the variability of epitopes within a given set of protein sequences. The tool was implemented as a component of the Immune Epitope Database and Analysis Resources (IEDB), and is directly accessible at http://tools.immuneepitope.org/tools/ conservancy.

Conclusion: An epitope conservancy analysis tool was developed to analyze the variability or conservation of epitopes. The tool is user friendly, and is expected to aid in the design of epitopebased vaccines and diagnostics.

\section{Background}

An epitope can be defined as a group of amino acids derived from a protein antigen that interacts with antibodies or T-cell receptors, thereby activating an immune response. Epitopes can be classified as either continuous or discontinuous. Continuous epitopes, also known as linear or sequential epitopes, are composed of amino acid residues that are contiguous in their primary protein sequence. Conversely, discontinuous epitopes, also known as assembled or conformational epitopes, are composed of amino acid residues that are typically present in different protein regions, but which are brought together by protein folding. Recognition of T cell epitopes typically depends upon processing of antigenic proteins, and as a result $\mathrm{T}$ cell epitopes are usually continuous. B cell epitopes, often recognized in the native protein context, may be either continuous or discontinuous.

Pathogenic proteins, in general, and epitopes in particular, are often variable. The degree of variability or similar- 
ity of specific proteins or protein regions can provide important information regarding evolutionary, structural, functional, and immunological correlates. Given a set of homologous proteins, phylogenetic relationships can be constructed and used to calculate the evolutionary rate at each amino acid site. Regions that evolve slowly are considered "conserved" while those that evolve rapidly are considered "variable". This approach is widely used in sequence conservation identification and mapping programs such as ConSeq [1] and ConSurf [2,3]. However, to fully describe and characterize protein and/or epitope variability, measures of identity and conservancy are typically utilized. Identity refers to the extent to which two amino acid sequences are invariant, and is measured as the percentage of identical amino acids in the alignment of two sequences. Conservancy is defined as the fraction of protein sequences that contain the epitope considered at or above a specified level of identity. Conversely, the fraction of protein sequences that contain the epitope considered below a specified level of identity reflects the degree of variability or uniqueness of the epitope.

Amino acid residues that are crucial for retention of protein function are believed to be associated with intrinsically lower variability, even under immune pressure. As such, these regions often represent good targets for the development of epitope-based vaccines, as the epitopes targeted can be expected to be present irrespective of disease stage, or particular strain of the pathogen. Furthermore, these same residues are often highly conserved across different related species, such as, for example, has been found in several instances in the context of the poxviridae [4]. As a result, a vaccine containing such conserved epitopes might be effective in providing broadspectrum protection. Conversely, in a diagnostic and disease monitoring setting, epitopes that are specific to a given pathogen can be used to monitor responses to that particular infectious strain, removing the confounding influence of immune responses derived from previous exposures to partially cross-reactive strains or organisms.

Herein, to assist in the selection of epitopes having a desired level of conservation or, conversely, variability, we have developed an epitope conservancy analysis tool. The tool has been specifically designed to determine the degree of conservation or variability associated with a specific epitope within a given set of protein sequences. Despite our emphasis on epitope identification contexts, it is also apparent that the tool can be utilized for other purposes, such as tracking mutation of epitopes during disease progression. This tool was implemented as a component of the Immune Epitope Database and Analysis Resources (IEDB) [5-7] and was used in predicting the cross-reactivity of influenza A epitopes [8].

\section{Implementation Approach}

Given an epitope sequence $\boldsymbol{e}$ and a set $\boldsymbol{P}$ of protein sequences $\{\boldsymbol{p}\}$, our approach is to find the best local alignment(s) of $\boldsymbol{e}$ on each $\boldsymbol{p}$. The degree of conservation of $\boldsymbol{e}$ within $\boldsymbol{P}$ is calculated as the fraction of $\{\boldsymbol{p}\}$ that matched the aligned $\boldsymbol{e}$ above a chosen identity level. Two separate processes were developed for assessing the degree of conservation/variability of continuous and discontinuous epitope sequences.

\section{Continuous sequence}

If $\boldsymbol{e}$ is continuous, the process of finding the best alignment of $\boldsymbol{e}$ on $\boldsymbol{p}$ involves breaking $\boldsymbol{p}$ in to sub-sequences $\{s\}$ of length equal to $\boldsymbol{e}$ and comparing $\boldsymbol{e}$ to each $\boldsymbol{s}$. For a $\boldsymbol{p}$ sequence of length $n$ and an $e$ sequence of length $m$, a total $n-m+1\{s\}$ different sequences are generated. For each $\boldsymbol{e}$ and $\boldsymbol{s}$ comparison, the degree of identity is calculated as a percent of residues that are identical between the two sequences. If $p$ contains repeat regions, or the identity threshold is low, multiple alignments may be found for $\boldsymbol{e}$. However, the $s$ sequence(s) associated with the maximum identity score determines the alignment(s) of $\boldsymbol{e}$ on $\boldsymbol{p}$. The degree of conservation of $\boldsymbol{e}$ is then calculated as the percent of $p$ sequences in which $e$ is aligned with an identity level at or above a chosen threshold. Conversely, the degree of variability is calculated as the fraction of $p$ that $\boldsymbol{e}$ was aligned below a chosen threshold. An illustrative conservancy analysis of a continuous epitope sequence is shown in Table 1.

\section{Discontinuous sequence}

If $\boldsymbol{e}$ is discontinuous, a continuous sequence pattern $c$ is first generated. For example, given a discontinuous sequence "A1, B3, C6" (meaning $\mathrm{A}$ is at position 1, B is at position 3 and $C$ is at position 6 ), its matching sequence pattern $c$ is AXBXXC where $\mathrm{X}$ is any amino acid residue, and the number of $X$ 's between two nearest known amino acid residues is equal to the gap distance between them. Next, the same procedure described for continuous sequences is used to identify the best alignment(s) of $c$ on $p$. The identity level is calculated based on the defined epitope residues. An illustration of a discontinuous sequence conservancy analysis is shown in Table 2. To obtain meaningful results, the program only performs calculations for discontinuous sequences consisting of at least three identified residues.

\section{Program description}

The epitope conservancy analysis tool was implemented as a Java web-application. An overview of the tool is shown in Figure 1. As input, the program requires the user to provide an epitope set, consisting of one or more epitope sequences, and a set of protein sequences against which each epitope is compared to determine conserv- 
Table I: Example conservancy analysis of a continuous sequence

\begin{tabular}{|c|c|c|c|c|c|c|c|c|c|c|c|c|}
\hline \multirow[b]{2}{*}{ Source } & \multicolumn{10}{|c|}{ Reference sequencel } & \multicolumn{2}{|c|}{ Identity ${ }^{2}$} \\
\hline & $F$ & L & $P$ & $S$ & $\mathrm{D}$ & $\mathrm{F}$ & $\mathrm{F}$ & $\mathrm{P}$ & $\mathrm{S}$ & V & No. & $(\%)$ \\
\hline Strain I & $\mathbf{Y}$ & L & $P$ & $S$ & $\mathrm{D}$ & $\mathrm{F}$ & $\mathrm{F}$ & $\mathrm{P}$ & $S$ & I & 8 & $(80)$ \\
\hline Strain 2 & $\mathrm{~F}$ & L & $P$ & $S$ & $\mathrm{D}$ & $\mathrm{F}$ & $\mathrm{F}$ & $\mathrm{P}$ & $\mathrm{S}$ & V & 10 & $(100)$ \\
\hline Strain 3 & $\mathbf{R}$ & $\mathrm{L}$ & $P$ & $S$ & $\mathbf{K}$ & $\mathbf{Q}$ & $\mathrm{F}$ & $\mathrm{P}$ & $\mathrm{S}$ & V & 7 & $(70)$ \\
\hline Strain 4 & $\mathbf{Y}$ & $\mathbf{E}$ & $P$ & $\mathbf{T}$ & $\mathrm{D}$ & $\mathrm{F}$ & $\mathrm{F}$ & $\mathrm{P}$ & $\mathrm{S}$ & V & 7 & (70) \\
\hline Strain 5 & $\mathrm{~F}$ & $\mathrm{~L}$ & $P$ & $\mathbf{T}$ & $\mathrm{D}$ & $\mathrm{F}$ & $\mathrm{F}$ & $\mathrm{P}$ & $\mathrm{S}$ & V & 9 & (90) \\
\hline Strain 6 & $\mathrm{~F}$ & $\mathrm{~L}$ & $P$ & $\mathbf{T}$ & $\mathrm{D}$ & $\mathrm{F}$ & $\mathbf{S}$ & $\mathbf{F}$ & $\mathbf{T}$ & V & 6 & $(60)$ \\
\hline Strain 7 & $\mathrm{~F}$ & $\mathrm{~L}$ & $P$ & $S$ & $D$ & $\mathrm{~F}$ & $\mathrm{~F}$ & $\mathrm{P}$ & $\mathrm{S}$ & V & 10 & $(100)$ \\
\hline Strain 8 & $\mathbf{Y}$ & $\mathbf{E}$ & $P$ & $S$ & $\mathbf{E}$ & $\mathrm{F}$ & $\mathbf{S}$ & $\mathbf{F}$ & $\mathrm{S}$ & I & 4 & (40) \\
\hline Strain 9 & $\mathrm{~F}$ & $\mathrm{~L}$ & $P$ & $S$ & $\mathbf{E}$ & $\mathrm{F}$ & $\mathrm{F}$ & $\mathrm{P}$ & $S$ & V & 9 & $(90)$ \\
\hline Strain 10 & $F$ & L & $P$ & $S$ & $D$ & $\mathrm{~F}$ & $\mathrm{~F}$ & $P$ & $S$ & V & 10 & $(100)$ \\
\hline \multicolumn{11}{|c|}{ Total: conservancy at identity threshold $\geq 80 \%$} & 6 & $(60)$ \\
\hline \multicolumn{11}{|c|}{ Total: variability at identity threshold $<80 \%$} & 4 & (40) \\
\hline
\end{tabular}

I. Residues that are different from that of the corresponding residue in the reference sequence are highlighted in bold.

2. Identity indicates the number (\%) of residues in the homologous sequence that are identical to the corresponding residue in the reference sequence.

3. Totals indicate the number (\%) of strains in which the reference sequence is found with an identity above or below the indicated threshold.

ancy. Based on our experience, to achieve the best results it is recommended that the protein sequence set utilized be constructed such that redundancies are eliminated and the representation of different substrains and serotypes is balanced. To assist in assembling protein sequence sets, a "Browse for sequences in NCBI" link is provided. When this link is selected, a browser is opened, enabling the user to search for all available protein sequences in NCBI, grouped by organism taxonomic level. To reduce redundancies in the protein sequence set, the user can check the box at the bottom of the input form to have the program automatically remove all duplicated sequences in the protein data set used in the analysis. As output, the program will calculate the fraction of protein sequences that match each epitope sequence above or below a given identity level. The program also calculates the minimum and maximum matching identity level for each epitope. A position mapping of epitope sequences to matching protein subfragments is also provided and can be viewed by clicking on the "Go" link in the "View details" column. Detailed

Table 2: Example conservancy analysis of a discontinuous sequence

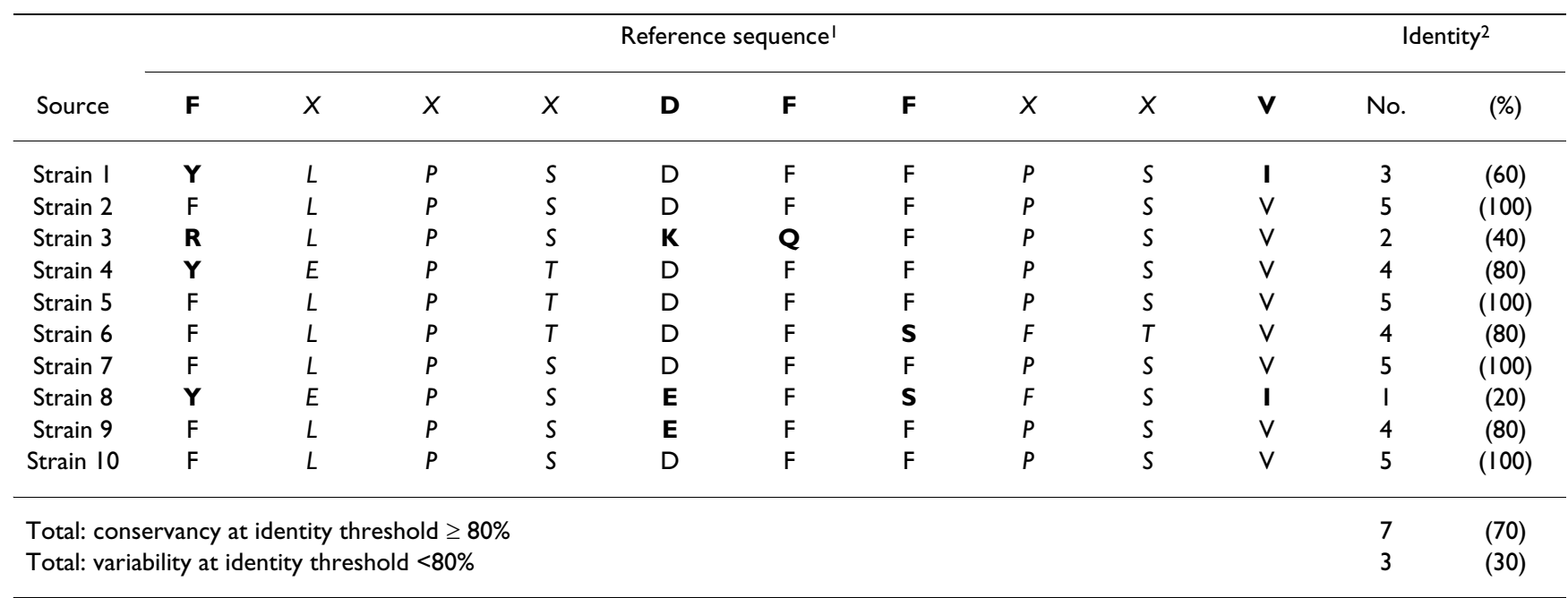

I. Residues that are not defined in the reference sequence are highlighted in italics. Residues that are different from that of the corresponding residue in the reference sequence are highlighted in bold.

2. Identity indicates the number (\%) of residues in the homologous sequence that are identical to the corresponding residue in the reference sequence. Residues highlighted with gray shading are not considered in calculating the identity because they are not defined in the reference sequence.

3. Totals indicate the number (\%) of strains in which the reference sequence is found with an identity above or below the indicated threshold. 


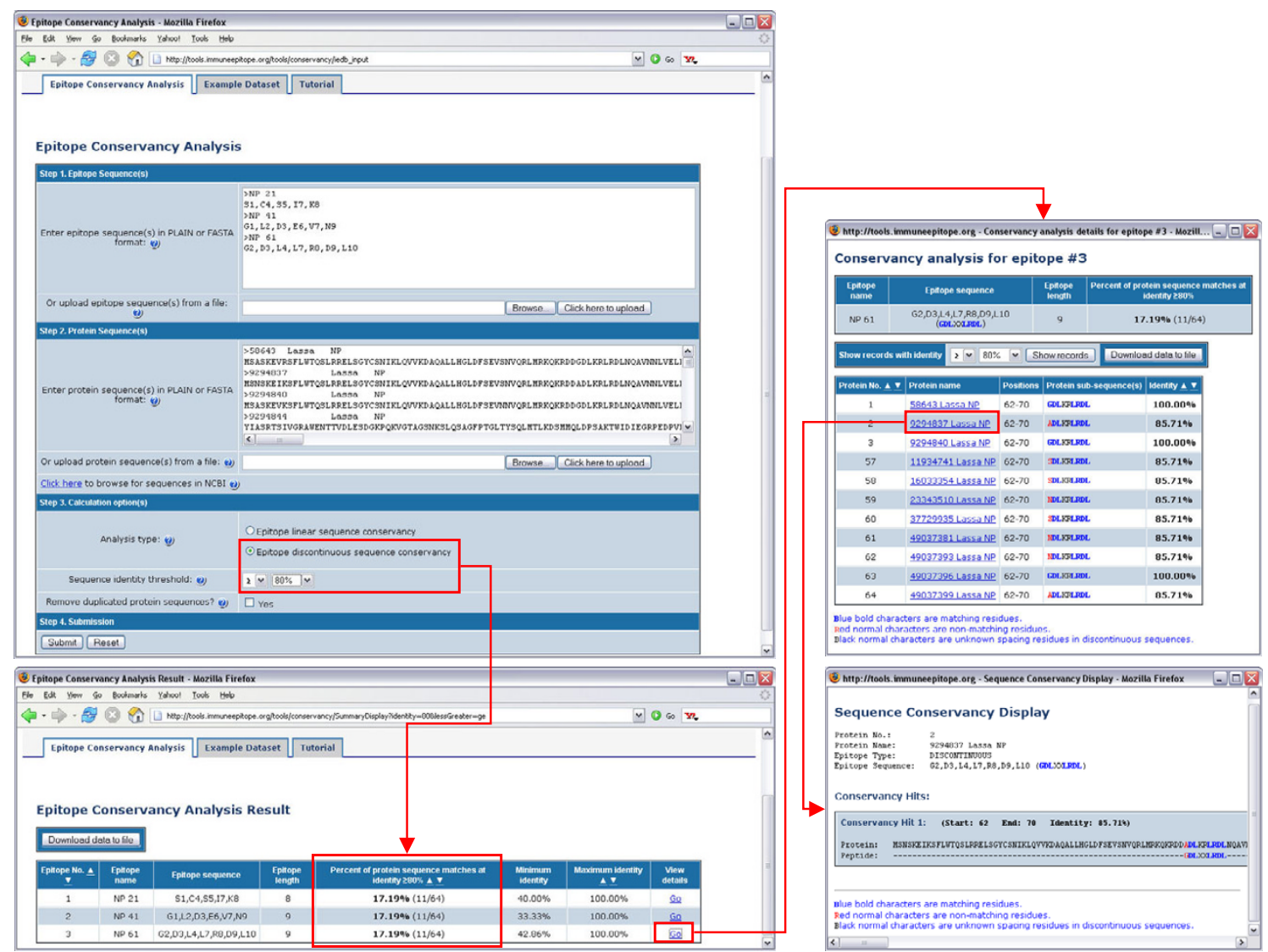

\section{Figure I}

An overview of the epitope conservancy analysis tool.

sequence mappings of an epitope to all protein sequences in a dataset are also generated. In some cases, if a protein sequence has significant repeat regions, or the level of matching identity is set at a low value, multiple matching protein sub-fragments can be found for a given epitope sequence. All calculation results can be downloaded as text files by clicking on the "Download data to file" button.

\section{Results and discussion}

To determine the degree of conservation of an epitope within a given set of protein sequences, it is necessary to align the epitope to each protein sequence. The degree of conservation is then calculated as the fraction of protein sequences that match the aligned epitope sequence above a defined identity level. Conversely, the degree of variablity is calculated as the fraction of protein sequences that match the aligned epitope sequence below a defined identity level. For continuous epitopes, existing sequence searching and alignment tools, such as BLAST [9] or Clus-
talW [10], can be used to perform pair-wise local alignment of the epitope to a protein sequence. But, to be relevant in an immunological context, it is crucial that the entire epitope sequence is completely aligned with absolutely no gaps. This requirement entails the use of somewhat different parameters making it cumbersome to use currently existing alignment tools for the characterization of immune epitopes. At the same time, there is no alignment tool currently available for analyzing discontinuous sequences. To rectify these shortcomings, we have developed a robust, user-friendly, epitope conservancy analysis tool. The tool has the capacity to simultaneously align and assess the degree of conservation/variability of each epitope, and can perform these functions for both linear and discontinuous peptide epitope sequences.

For the purpose of developing cross-reactive vaccines that aim toward highly variable pathogens, the use of conserved epitopes across different species is desired. Nevertheless, care should be taken to avoid selecting epitopes 
that are conserved between the pathogen and the host as this could lead to undesirable induction of auto-immunity. Moreover, extremely conserved epitopes between species are sometimes less immunogenic because they may be derived from proteins that resemble similar proteins in the host. As a result, they are less likely to be recognized by $\mathrm{T}$ cells due to self-tolerance. It should also be emphasized that conservation at the sequence level does not assure that the epitope will be equally recognized and cross-reactive. This is due to the differences in the antigen sequences from which the epitope is derived. For $\mathrm{T}$ cell epitopes, whether they will be processed in the first place is determined by flanking residues that are different for different antigens. Therefore, the same epitope sequence from different antigens may or may not be generated to subsequently presented and recognized by $\mathrm{T}$ cell receptors.

In the case of B cell epitopes, their recognition by an antibody is dependent on the antigen 3D structures. A sequence-wise conserved epitope may not be structurally conserved as it can adopt different conformations in the context of the antigen structures. Exposed amino acids as opposed to buried amino acids are more important in determining the immunogenic of a given segment of peptide. It is because only exposed residues, as observed in antigen:antibody co-crystals, can form contacts with the complementarity determining regions (CDRs) of the corresponding antibody. Those residues that are recognized by a single antibody are often defined as a discontinuous epitope. The epitope conservancy analysis tool developed here can be used to assess the pattern conservation of discontinuous epitopes. Nevertheless, pattern-wise conserved discontinuous epitopes may not be cross-reactive due to the unknown influence of neighboring and interdispersed amino acids. As a result, if antigen structures are available, it may be better to predict cross-reactivity based on the epitope's 3D structural conservation.

Depending on the specific needs of a user, an analysis of epitope conservancy may need to be performed at various phylogenetic levels. For example, to determine the potential of a given epitope to be cross-reactive amongst different isolates of a pathogen, or with different microorganisms associated with different pathogenicity, it may be necessary to determine conservancy within a given sub strain, type or clad, within a specific species, or within a genus, or other higher phylogenetic classification group. This type of analysis was utilized previously to identify highly conserved HBV derived epitopes $[11,12]$, and also applied to identify HCV, P. falciparum and HIV derived epitopes [[13], [14], [15], [16], [17], [18], [19]]. Alternatively, to develop epitope-based diagnostic applications aimed at detecting all isolates of a given pathogen but not isolates from related strains, or aimed at detecting specific strains or isolates, it might be necessary to identify epitopes that are highly conserved in only a single or just a few isolates, and poorly conserved in others. Finally, the analysis of potential homologies with sequences expressed by a pathogen's host, or an animal species to be used as an animal model, might be of particular relevance. We anticipate that its relevance might range from predicting poor responses due to self-tolerance and differential performance in animal species expressing different degrees of similarities with a given epitope, to predicting potential safety problems and autoreactivity linked to cross-reactive self reactivity and molecular mimicry. For each of these broad applications, the analysis tool we have developed provides the means to easily assemble the protein sets required to undertake the appropriate analyses, and generates the information necessary to make the appropriate design decisions.

\section{Conclusion}

To address the issue of conservation (or variability) of epitopes or, more broadly speaking, peptide sequences, we have developed a tool to calculate the degree of conservancy (or inversely, the variability) of an epitope within a given protein sequence set. Conservancy can be calculated following user defined identity criteria, and minimal and maximal levels of conservancy are identified. Furthermore, the program provides detail information for each alignment executed. This epitope conservancy analysis tool is publicly available and can be used to assist in the selection of epitopes with the desired pattern of conservation for designing epitope-based diagnostics and vaccines.

\section{Availability and requirements \\ - Project name: Epitope Conservancy Analysis}

- Project home page: http://tools.immuneepitope.org/ tools/conservancy

- Operating system(s): Platform independent

- Programming language: Java

- Other requirements: Java 1.4 or higher, Tomcat 4.0 or higher

- License: none

- Any restrictions to use by non-academics: none

Abbreviations

BLAST: Basic Local Alignment Search Tool

CDRs: Complementarity determining regions 
IEDB: Immune Epitope Database and Analysis Resources

MSA: Multiple sequence alignment

NCBI: National Center for Biotechnology Information

\section{Competing interests}

The author(s) declares that there are no competing interests.

\section{Authors' contributions}

HHB developed the program. WL and NF participated in programming tasks. HHB, JS and AS wrote the manuscript. All authors read and approved the final version.

\section{Acknowledgements}

The authors would like to thank two anonymous reviewers for their constructive suggestions. This work was supported by the National Institutes of Health's contract HHSN26620040006C (Immune Epitope Database and Analysis Program) and generous support from Gemini, Kirin pharmaceutical division. This is LIAI publication number 823 .

\section{References}

I. Berezin C, Glaser F, Rosenberg J, Paz I, Pupko T, Fariselli P, Casadio $\mathrm{R}$, Ben-Tal N: ConSeq: the identification of functionally and structurally important residues in protein sequences. Bioinformatics 2004, 20(8): I322-I324.

2. Glaser F, Pupko T, Paz I, Bell RE, Bechor-Shental D, Martz E, Ben-Tal $\mathrm{N}$ : ConSurf: identification of functional regions in proteins by surface-mapping of phylogenetic information. Bioinformatics 2003, 19(1):163-164.

3. Landau M, Mayrose I, Rosenberg Y, Glaser F, Martz E, Pupko T, BenTal N: ConSurf 2005: the projection of evolutionary conservation scores of residues on protein structures. Nucleic Acids Res 2005:W299-302

4. Pasquetto V, Bui HH, Giannino R, Mirza F, Sidney J, Oseroff C, Tscharke DC, Irvine K, Bennink JR, Peters B, Southwood S, Cerundolo V, Grey H, Yewdell JW, Sette A: HLA-A*020I, HLA-A*I I II, and HLA-B*0702 Transgenic Mice Recognize Numerous Poxvirus Determinants from a Wide Variety of Viral Gene Products. J Immunol 2005, 175(8):5504-55I5.

5. IEDB [http://www.immuneepitope.org]

6. Peters B, Sidney J, Bourne P, Bui HH, Buus S, Doh G, Fleri W, Kronenberg $M$, Kubo R, Lund O, Nemazee D, Ponomarenko JV, Sathiamurthy M, Schoenberger S, Stewart S, Surko P, Way S, Wilson S, Sette A: The immune epitope database and analysis resource: from vision to blueprint. PLoS Biol 2005, 3(3):e9I.

7. Peters B, Sidney J, Bourne P, Bui HH, Buus S, Doh G, Fleri W, Kronenberg M, Kubo R, Lund O, Nemazee D, Ponomarenko JV, Sathiamurthy M, Schoenberger SP, Stewart S, Surko P, Way S, Wilson S, Sette A: The design and implementation of the immune epitope database and analysis resource. Immunogenetics 2005, 57(5):326-336.

8. Bui HH, Peters B, Assarsson E, Mbawuike I, Sette A: Ab and T cell epitopes of influenza $A$ virus, knowledge and opportunities. Proc Natl Acad Sci USA 2007, I04(I):246-25I.

9. Altschul SF, Madden TL, Schaffer AA, Zhang J, Zhang Z, Miller W, Lipman DJ: Gapped BLAST and PSI-BLAST: a new generation of protein database search programs. Nucleic Acids Res 1997, 25(I7):3389-3402.

10. Thompson JD, Higgins DG, Gibson TJ: CLUSTAL W: improving the sensitivity of progressive multiple sequence alignment through sequence weighting, position-specific gap penalties and weight matrix choice. Nucleic Acids Res 1994, 22(22):4673-4680.

II. Bertoni R, Sidney J, Fowler P, Chesnut RW, Chisari FV, Sette A: Human histocompatibility leukocyte antigen-binding supermotifs predict broadly cross-reactive cytotoxic $T$ lym- phocyte responses in patients with acute hepatitis. J Clin Invest 1997, I00(3):503-5।3.

12. Cerny A, Ferrari C, Chisari FV: The class I-restricted cytotoxic $\mathbf{T}$ lymphocyte response to predetermined epitopes in the hepatitis B and C viruses. Curr Top Microbiol Immunol 1994, 189:169-186.

13. Doolan DL, Hoffman SL, Southwood S, Wentworth PA, Sidney J, Chesnut RW, Keogh E, Appella E, Nutman TB, Lal AA, Gordon DM, Oloo A, Sette A: Degenerate cytotoxic $\mathbf{T}$ cell epitopes from $\mathbf{P}$. falciparum restricted by multiple HLA-A and HLA-B supertype alleles. Immunity 1997, 7(I):97-II 2.

14. Doolan DL, Southwood S, Chesnut R, Appella E, Gomez E, Richards A, Higashimoto YI, Maewal A, Sidney J, Gramzinski RA, Mason C, Koech D, Hoffman SL, Sette A: HLA-DR-promiscuous T cell epitopes from Plasmodium falciparum pre-erythrocyticstage antigens restricted by multiple HLA class II alleles. J Immunol 2000, 165(2): I I23-1 I37.

15. Wilson CC, Palmer B, Southwood S, Sidney J, Higashimoto Y, Appella $E$, Chesnut $R$, Sette A, Livingston BD: Identification and antigenicity of broadly cross-reactive and conserved human immunodeficiency virus type I-derived helper T-lymphocyte epitopes. J Virol 200I, 75(9):4I95-4207.

16. Chang KM, Gruener NH, Southwood S, Sidney J, Pape GR, Chisari FV, Sette A: Identification of HLA-A and -B7-restricted CTL response to hepatitis $C$ virus in patients with acute and chronic hepatitis C. J Immunol 1999, I 62(2): I I56-I I64.

17. Lamonaca V, Missale G, Urbani S, Pilli M, Boni C, Mori C, Sette A, Massari M, Southwood S, Bertoni R, Valli A, Fiaccadori F, Ferrari C: Conserved hepatitis $C$ virus sequences are highly immunogenic for CD4(+) T cells: implications for vaccine development. Hepatology 1999, 30(4): 1088-1098.

18. Altfeld MA, Livingston B, Reshamwala N, Nguyen PT, Addo MM, Shea A, Newman M, Fikes J, Sidney J, Wentworth P, Chesnut R, Eldridge RL, Rosenberg ES, Robbins GK, Brander C, Sax PE, Boswell S, Flynn T, Buchbinder S, Goulder PJ, Walker BD, Sette A, Kalams SA: Identification of novel HLA-A2-restricted human immunodeficiency virus type I-specific cytotoxic T-lymphocyte epitopes predicted by the HLA-A2 supertype peptide-binding motif. J Virol 200I, 75(3): I30I-I3|I.

19. Wentworth PA, Sette A, Celis E, Sidney J, Southwood S, Crimi C Stitely S, Keogh E, Wong NC, Livingston B, Alazard D, Vitiello A, Grey HM, Chisari FV, Chesnut RW, Fikes J: Identification of A2restricted hepatitis C virus-specific cytotoxic $\mathbf{T}$ lymphocyte epitopes from conserved regions of the viral genome. Int Immunol 1996, 8(5):65I-659.

Publish with Bio Med Central and every scientist can read your work free of charge

"BioMed Central will be the most significant development for disseminating the results of biomedical research in our lifetime. "

Sir Paul Nurse, Cancer Research UK

Your research papers will be:

- available free of charge to the entire biomedical community

- peer reviewed and published immediately upon acceptance

- cited in PubMed and archived on PubMed Central

- yours - you keep the copyright
BioMedcentral 